\title{
Mutual Localization using Anonymous Bearing Measurements
}

\author{
Paolo Stegagno, Marco Cognetti, Antonio Franchi, Giuseppe Oriolo
}

\begin{abstract}
This paper addresses the problem of mutual localization in multi-robot systems in presence of anonymous (i.e., without the identity information) bearing-only measurements. The solution of this problem is relevant for the design and implementation of any decentralized multi-robot algorithm/control. A novel algorithm for probabilistic multiple registration of these measurements is presented, where no global localization, distances, or identity are used. With respect to more conventional solutions that could be conceived on the basis of the current literature, our method is theoretically suitable for tasks requiring frequent, many-to-many encounters among agents (e.g., formation control, cooperative exploration, multiple-view environment sensing). An extensive experimental study validates our method and compares it with the fullinformative case of bearing-plus-distance measurements. The results show that the proposed localization system exhibits an accuracy commensurate to our previous method [1] which uses bearing-plus-distance information.
\end{abstract}

\section{INTRODUCTION}

Consider a system of moving agents, each equipped with its own attached frame. Relative Mutual Localization (RML) is the problem of estimating the relative poses among the agents' frames. Alternatively, each agent can use its own fixed frame to express poses, leading to Absolute Mutual Localization (AML) as the problem of estimating the relative poses among the various fixed frames. The two problems are clearly equivalent provided that each agent is localized w.r.t. its own fixed frame.

Mutual localization is essential for achieving decentralization, and hence autonomy and robustness, in multiagent applications, such as consensus [2], flocking [3], exploration [4], formation control [5], connectivity maintenance [6], distributed estimation [7], cooperative transportation [8], coverage and sensing [9], [10].

In the literature, one finds a certain number of works dealing with the localization of static agents, commonly referred to as network localization [11], [12], [13], [14], [15]. Other papers have considered moving agents, with the objective of estimating their poses in a common fixed frame [16], [17], [18], [19], [20], and have generally shown that the agents' ability of sensing each other can be used to improve the localization of the entire system. In the literature, this kind of approach called cooperative localization. Note that agreeing on a common fixed frame already implies a centralized consensus.

Most of the abovementioned works assume that the relative measurements come with the identity of the involved

P. Stegagno, M. Cognetti and G. Oriolo are with DIS, Università di Roma La Sapienza, 00185 Rome, Italy (e-mail: stegagno@dis.uniroma1.it, marco.cognetti@gmail.com,oriolo@ dis.uniroma1.it).

A. Franchi is with the Max Plank Institute for Biological Cybernetics, 72012 Tübingen, Germany (e-mail: antonio.franchi@tuebingen.mpg.de). agents. This requires some form of agent tagging which depends on the specific sensor. However, the definition of an identity tagging is in itself a form of centralization. Moreover, tagging and identification may be difficult in large groups, especially when low-information sensors such as range finders are used, but even with potentially richer sensors such as video cameras. Therefore, we argue that the study of mutual localization using anonymous (i.e., without identity information) measurements is relevant for the design of completely decentralized systems.

In our previous works, we studied the impact of anonymity on mutual localization problems using position measurements. In [21], we proposed an AML/RML system consisting of two components: (1) MultiReg, a multiple registration algorithm that computes the change of coordinates among the relative frames of the agents using geometrical arguments to invert the measurement map (2) MH-EKF, a multi-hypothesis Extended Kalman Filter that uses the output of MultiReg and the motion displacements to estimate the change of coordinates among the fixed frames of the agents. In [22] we have shown that anonymity causes a combinatorial ambiguity in the geometrical computation of coordinate changes when the formation is rotational symmetric. In this case, or even when the formation is 'almost' symmetric, the complexity of the MultiReg algorithm becomes exponential with the number of agents. Since in many applications (e.g., entrapment or escorting) multi-agent systems are required to move in regular, possibly rotational symmetric formations, this worstcase complexity may indeed materialize, severely affecting the performance of localization systems based on MultiReg. In [1], we have significantly modified the localization system of [21] in a probabilistic sense to overcome the aforementioned difficulties. The key ideas are (1) to use a particle filter to directly estimate the probability function of the agents' relative poses (in other words, to solve RML also in the filter) and (2) to modify MultiReg so that is can use this information as a feedback.

In this paper, we address the RML problem using anonymous bearing-only measurements rather than full position (bearing plus distance) measurements. This represents the practical situation in which the agents are equipped with non-depth sensors such as video cameras. The literature on bearing-only localization (e.g., see [12], [23], [24]) focuses essentially on the non-anonymous case; in particular, in [25] it has been shown that the system is observable in presence of bearing-only and odometry measurements.

With respect to the anonymous position measurement case considered in [1], the use of anonymous bearing-only measurements is undoubtedly more challenging, mainly for two reasons. First, the number of possible solutions due to 
the anonymity increases in general, because the measurements provide less information content. Second, the scale information (i.e., the relative distance) is missing from the measurement and must be reconstructed by appropriately fusing the exteroceptive sensor information (the bearing angles) with the odometric data.

The paper is organized as follows. The formulation of the problem is given in Sect. II. The proposed mutual localization system is described in Sect. III, and validated via simulations and experiments in Sect. IV. Possible extensions of this work are sketched in Sect. V.

\section{PROBLEM FORMULATION}

Throughout this section, refer to Fig. 1 for illustration. Consider $n$ planar agents (called robots from now on) $\mathcal{R}_{1}, \ldots, \mathcal{R}_{n}$, with $n$ unknown and possibly changing over time. Let $\mathcal{N}=\{1, \ldots, n\}$ and $\mathcal{N}_{k}=\mathcal{N} /\{k\}$. A moving frame $\mathcal{F}_{k}$ is attached to each $\mathcal{R}_{k}$.

Consider the generic robot $\mathcal{R}_{k}, k \in \mathcal{N}$. We assume that it is equipped with a motion detector (or odometer) that measures $u_{k}^{t}$, the robot displacement between two consecutive sampling instants $t-1$ and $t$. The motion detector is characterized by a probabilistic motion model $p\left(u^{\prime} \mid u\right)$, where $u^{\prime}$ and $u$ are, respectively, the true and the measured displacement.

In addition, $\mathcal{R}_{k}$ carries a robot detector, a sensor device that detects other robots within a perception set $D_{p}$ and measures their bearing angles w.r.t. $\mathcal{F}_{k}$, without the associated identity. This detector is prone to false positives (detected objects that look like robots) and false negatives (undetected robots, e.g., due to occlusions). In the following, bearing measurements will be generically referred to as features, to emphasize that they are anonymous and, in any case, may or not represent actual robots. Denote by $B_{k}^{t}$ the set of features seen by $\mathcal{R}_{k}$ at time $t$.

The equipment of $\mathcal{R}_{k}$ is completed by a communication module that can send/receive data to/from any other robot $\mathcal{R}_{h}, h \in \mathcal{N}_{k}$, contained in a communication set $D_{c}$. We assume that $D_{p} \subset D_{c}$, so that if $\mathcal{R}_{k}$ can detect $\mathcal{R}_{h}$ it can also communicate with it. Each message sent by $\mathcal{R}_{k}$ contains: (1) the robot signature (2) $v_{k}^{t}$, the total displacement of the robot between $t$ and 0 , computed by composing the elementary displacements $u_{k}^{1}, \ldots, u_{k}^{t}$ (3) the feature set $B_{k}^{t}$. Denote by $C_{k}^{t}$ the current set of neighbors, i.e., robots which are in communication with $\mathcal{R}_{k}$ at time $t$, and let $C_{k}^{1: t}=\cup_{\tau=1}^{t} C_{k}^{\tau}$.

Note that no probabilistic model is attached to the robot detector - instead, we will directly add uncertainty on the output of the multiple registration algorithm. Since the latter automatically removes false positives and negatives, we thus avoid the necessity of modeling the generation of these outliers. As for communication, we assume it to be ideal for simplicity; however, one may easily set up a probabilistic model to account for the uncertainty that affects this process.

From now on, consider the relative localization problem from the viewpoint of the generic robot $\mathcal{R}_{i}$. For $j \in \mathcal{N}_{i}$, let $x_{j}$ be the relative pose of $\mathcal{R}_{j}, j \in \mathcal{N}_{i}$, i.e., the 3 -vector describing the position and orientation of $\mathcal{F}_{j}$ w.r.t. $\mathcal{F}_{i}$; and let $\xi_{j}$ be its relative bearing-orientation, i.e., the 2 -vector

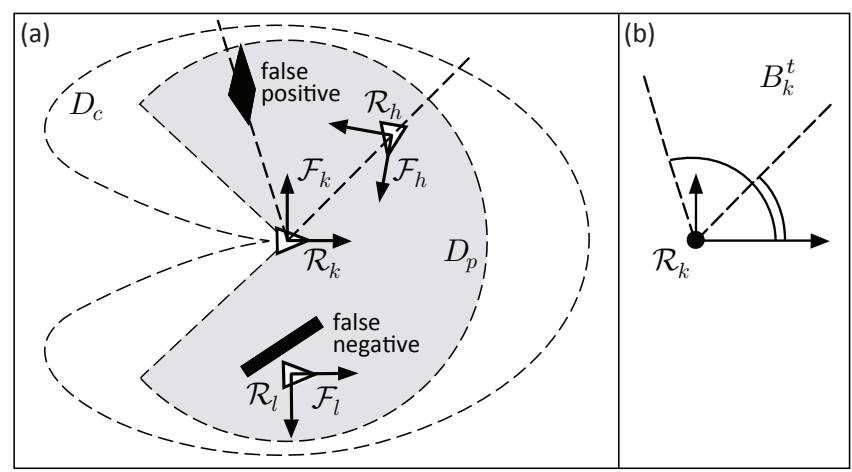

Fig. 1: Mutual localization with anonymous bearing measurements (a) A group of robots (white triangles) with the associated moving frames. Also shown are the perception and communications sets for robot $\mathcal{R}_{k}$; note how in this case the obstacles (black polygons) create one false positive and one false negative. (b) As a consequence, the feature set $B_{k}^{t}$ includes two anonymous bearing measurements.

containing the bearing angle and orientation of $\mathcal{F}_{j}$ w.r.t. $\mathcal{F}_{i}$. Note that $\xi_{j}$ can be considered as a partial representation of $x_{j}$ without the scale information. We shall use the wellknown operators $\oplus$ and $\ominus$ for the composition of poses.

In a probabilistic framework, solving the RML problem with anonymous bearing measurements requires $\mathcal{R}_{i}$ to compute its current belief about the relative poses of all the robots with which $\mathcal{R}_{i}$ has communicated, on the basis of the odometric and bearing measurements gathered directly by $\mathcal{R}_{i}$ or obtained via communication with other robots:

Problem 1: (Probabilistic RML with anonymous bearing measures) For $t=1,2, \ldots$ and $j \in C_{i}^{1: t}$, compute the following belief

$$
\operatorname{bel}\left(x_{j}^{t}\right)=p\left(x_{j}^{t} \mid u_{i}^{1: t}, B_{i}^{1: t},\left\{v_{j}^{\tau}, B_{j}^{\tau}\right\}_{j \in C_{i}^{\tau}, \tau=1, \ldots, t}\right)
$$

with the superscript $1: t$ denoting the complete history of a variable up to time $t$.

\section{PROPOSED APPROACH}

The mutual localization system we propose ${ }^{1}$ for the solution of Problem 1 is similar to that in [1], with the appropriate modifications to account for the availability of bearing (rather than position) measurements.

As shown in Fig. $2, \mathcal{R}_{i}$ first applies the multiple registration algorithm P-MultiBeaReg to compute the most 'likely' relative bearing-orientation of each robot belonging to $C_{i}^{t}$, based on the sets of features $B_{i}^{t}$ and $\left\{B_{j}^{t}\right\}_{j \in C_{i}^{t}}$, as well as on the current beliefs about $\left\{x_{j}^{t}\right\}_{j \in C_{i}^{t}}$. The relative bearingorientations thus obtained, together with the motion detector measures $u_{i}^{t}$ and $\left\{v_{j}^{t}\right\}_{j \in C_{i}^{t}}$, are used by particle filters to update the belief about the pose of each robot in $C_{i}^{1: t}$.

P-MultiBeaReg, which can be considered as the main contribution of this paper, is described in Sect. III-A. Since this algorithm only provides the relative bearing-orientation

\footnotetext{
${ }^{1}$ The reader is referred to [1] for a discussion of the potential advantages of this approach over more conventional solutions to the anonymity issue, e.g., based on Mahalanobis-distance data association. Essentially, we aim at designing a localization method that is effective in the presence of frequent and/or more-than-pairwise encounters among robots, typically found, e.g. in formation control or cooperative exploration.
} 


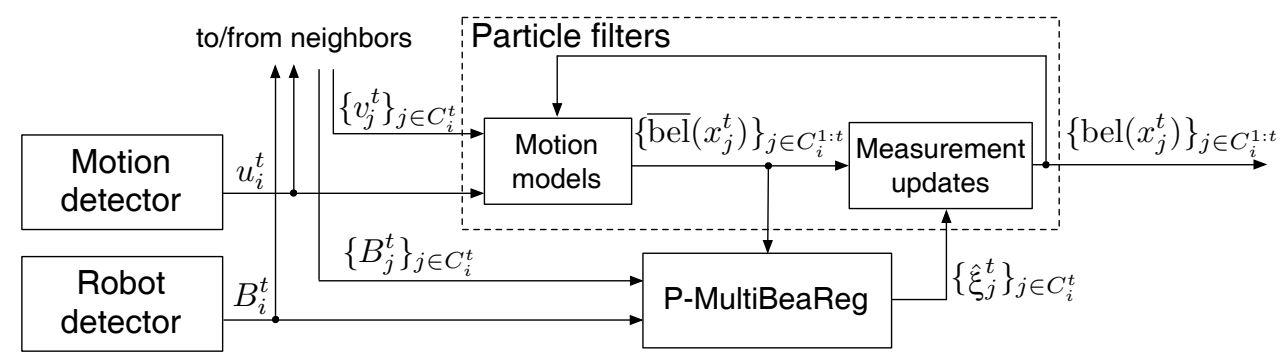

Fig. 2: Scheme of the mutual localization system that runs on $\mathcal{R}_{i}$.

of robots belonging to $C_{i}^{t}$, as opposed to the full relative poses provided by P-MultiReg in [1], minor adaptations are also needed in the design of the particle filters, briefly presented in Sect. III-B.

\section{A. Probabilistic Multiple Bearing Registration}

P-MultiBeaReg is a probabilistic multiple registration algorithm run by $\mathcal{R}_{i}$ at each time instant $t$ to feed the measurement update of the particle filters (see Fig. 2). In general, registration is the process of computing the relative pose between two or more different viewpoints of the same scene. In our case, since the 'scene' consists only of bearing angles, the scale of the relative pose cannot be recovered. In particular, given the sets of features $B_{i}^{t},\left\{B_{j}^{t}\right\}_{j \in C_{i}^{t}}$ and the current beliefs $\left\{\overline{\operatorname{bel}}\left(x_{j}^{t}\right)\right\}_{j \in C_{i}^{t}}$ computed in the particle filters through the motion model of the robots (see Fig. 2), P-MultiBeaReg derives an estimate $\hat{\xi}_{j}^{t}$ of the relative bearingorientation of $\mathcal{R}_{j}^{t}, j \in C_{i}^{t}$, w.r.t. $\mathcal{R}_{i}$.

A pseudo-code description of P-MultiBeaReg is given in Algorithm 1. The basic steps are illustrated in Fig. 3.

Consider the configuration of the multi-robot system shown in Fig. 3a, with the corresponding feature sets in Fig. 3b. Note that each pair of features (bearing angles) in the same feature set can be equivalently represented by

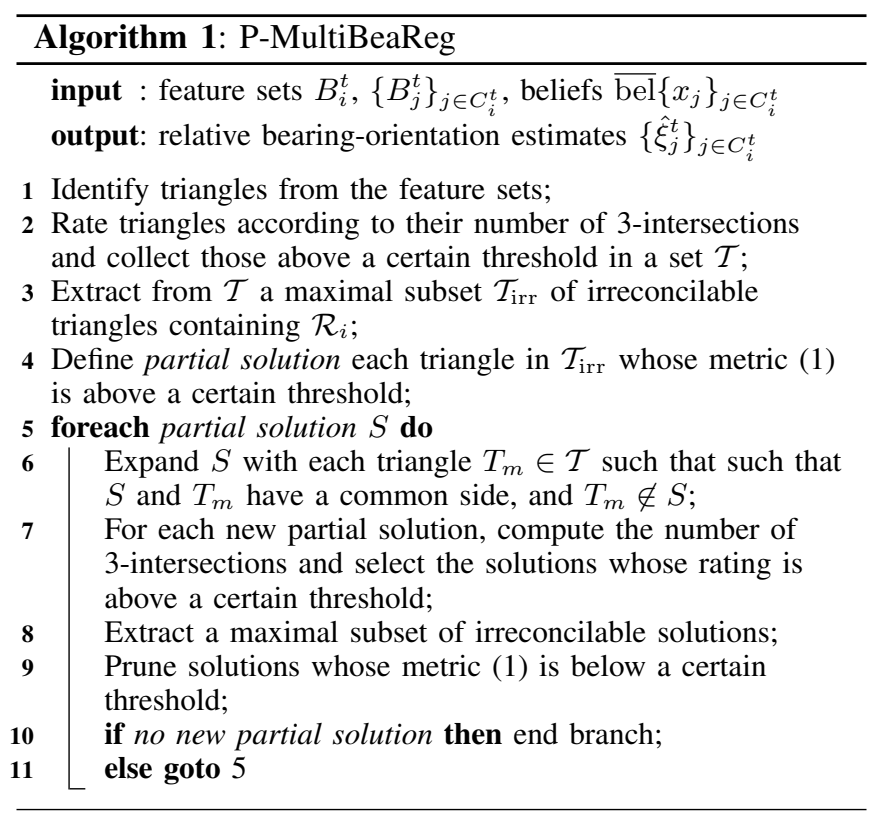

their difference angle. Now take a triplet of robots that 'see' each other, e.g., $\mathcal{R}_{i}, \mathcal{R}_{j}, \mathcal{R}_{k}$, and make $\mathcal{R}_{h}$ 'disappear' for a moment, so that each robot in the triplet sees only two features, or equivalently one difference angle; since the triplet defines a triangle, the sum of the three difference angles must be $\pi$. The algorithm exploits this basic observation by scanning all the possible triplets of feature sets and looking for triplets of difference angles (one from each feature set) whose sum is $\pi$, with a certain tolerance. Each of these triplets defines a triangle; more precisely, it defines a class of equivalence, because the triangle is defined only up to a scaling factor. Note that a triangle includes the identity of the robots at its vertices.

When three robots forming a triangle see a fourth robot (e.g., $\mathcal{R}_{h}$ in Fig. 3a), their sets of features include three rays (one from each feature set) that intersect in a single point (we call this a 3-intersection). Based on this idea, the algorithm rates all the triangles by counting their 3 -intersections ${ }^{2}$, and discards those below a certain threshold. From the remaining set $\mathcal{T}$, one extracts a maximal subset $\mathcal{T}_{\text {irr }}$ of irreconcilable triangles containing $\mathcal{R}_{i}$ (two triangles are irreconcilable if they associate the same robot to different features of the same set, or two different robots to the same feature).

The results of this process of triangle finding and rating are illustrated in Fig. 3c-f. In particular, Fig. 3c shows all the triangles having one 3 -intersection and containing $\mathcal{R}_{i}$; Fig. $3 \mathrm{e}$ shows all the triangles having one 3 -intersection but not containing $\mathcal{R}_{i}$; whereas Fig. $3 \mathrm{f}$ depicts one of the triangles without 3 -intersections. One choice for the maximal subset $\mathcal{T}_{\text {irr }}$ is shown in Fig. 3 d.

The next step is aimed at validating the triangles in $\mathcal{T}_{\text {irr }}$ on the basis of the current belief about the pose of the robots. To this end, we use the metric function

$$
P\left(\hat{x}_{j}^{t}\right)=\int p\left(\hat{x}_{j}^{t} \mid x_{j}^{t}\right) \overline{\operatorname{bel}}\left(x_{j}^{t}\right) d x_{j}^{t} .
$$

First, the scale of each triangle is computed so as to maximize the function, and then an adaptive thresholding of these maximum values is used to keep only the triangles that better fit the belief.

Each triangle surviving the previous step is used as initialization of a partial solution, and originates a branch of the algorithm aimed at iteratively expanding the partial solution with the addition of other triangles (see Fig. $3 \mathrm{~g}$ ).

\footnotetext{
${ }^{2}$ A simple 2-intersection does not provide any useful information, since two non-parallel rays will always intersect at a point.
} 


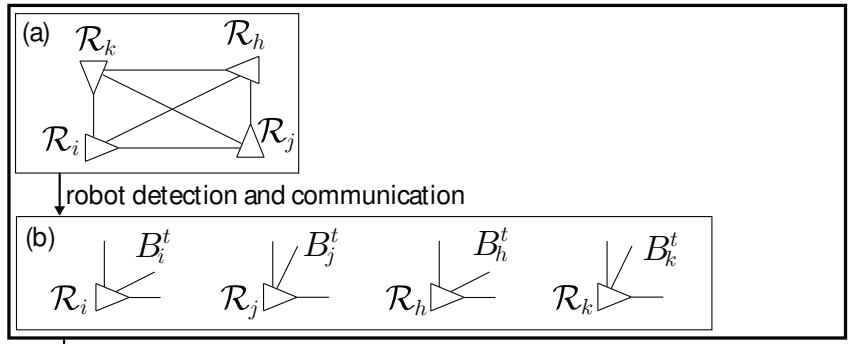

find and rate triangles

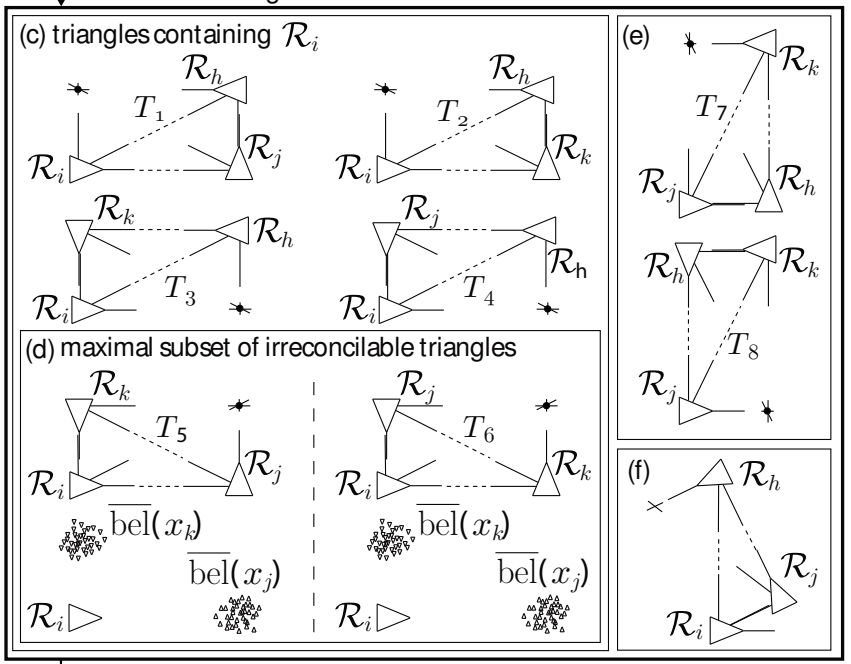

rating based expansion of the solutions

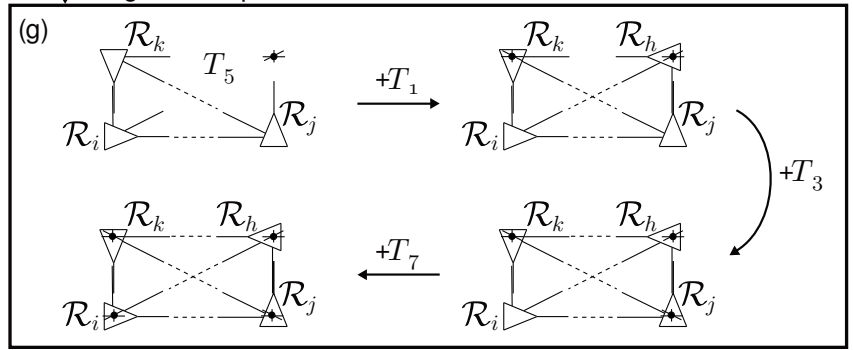

Fig. 3: Illustration of the basic steps of P-MultiBeaReg in a simple situation: (a) actual configuration (b) feature sets (c) all the triangles having one 3 -intersection and containing $\mathcal{R}_{i}(\mathrm{~d})$ one choice for the maximal subset $\mathcal{T}_{\text {irr }}$ and comparison with the current belief (e) all the triangles having one 3 -intersection but not containing $\mathcal{R}_{i}$ (f) one of the triangles without 3 -intersections $(\mathrm{g})$ expansion of the partial solution using the remaining triangles in $\mathcal{T}$.

In particular, let $S$ be the partial solution (a collection of triangles) associated to a branch at a given step. Denote by $\mathcal{T}_{S}=\left\{T_{S 1}, \ldots, T_{S M}\right\}$ the triangles in $\mathcal{T}$ that are not contained in $S$ and have one common side with $S$. The algorithm builds $M$ partial solutions by expanding $S$ with $T_{S i}, i=1, \ldots, M$. Each solution is then rated by counting its total number of 3 -intersections ${ }^{3}$. Then, the algorithm selects a subset of partial solutions whose rating is above a certain threshold, extracts from this set a maximal subset

\footnotetext{
${ }^{3}$ Note that two 3-intersections that match generate a 4-intersection, that counts as four 3 -intersections. In general, an $n$-intersection counts as $n ! /[(n-3) ! 3 !] 3$-intersections. A triangle vertex matching a 3-intersection counts as an additional 4-intersection; adding a triangle that is not in the solution but whose vertexes are already in the solution also counts as an additional 4-intersection.
}

of irreconcilable elements, and rates them using the metric function (1). Only the solutions which adequately fit the current belief according to an adaptive threshold are passed as partial solutions to following step, in which each solution originates a new branch of the algorithm. Each branch is expanded in an iterative process until the associated $\mathcal{T}_{S}$ becomes empty. The final solutions produced by all branches are then rated to identify the most likely relative bearingorientation estimates.

\section{B. Particle Filters}

The particle filters that complete our mutual localization system are similar to those in [1], with only few adaptations.

Robot $\mathcal{R}_{i}$ maintains one ${ }^{4}$ particle filter for each $\mathcal{R}_{j}$, $j \in C_{i}^{1: t}$. The inputs of the $j$-th filter at time $t$ are the displacement $u_{i}^{t}$ of $\mathcal{R}_{i}$, the total displacement $v_{j}^{t}=u_{j}^{1} \oplus \cdots \oplus$ $u_{j}^{t}$ of $\mathcal{R}_{j}$ (sent by $\mathcal{R}_{j}$ ) and the relative bearing-orientation estimate $\hat{\xi}_{j}^{t}$ (computed by P-MultiBeaReg). The latter is used to generate a gaussian measurement model with mean value $\hat{\xi}_{j}^{t}$ and appropriate covariance. If P-MultiBeaReg generates $m>1$ estimates (e.g., due to ambiguity), the model is given by the normalized sum of $m$ gaussians centered at the estimates.

The update rules accounting for the motion of $\mathcal{R}_{i}$ and $\mathcal{R}_{j}$ are respectively

$$
\begin{aligned}
& p\left(x_{j} \mid u_{i}\right)=N_{i} \int p\left(u^{\prime} \mid u_{i}\right) p\left(x_{j} \oplus u^{\prime}\right) d u^{\prime} \\
& p\left(x_{j} \mid u_{j}\right)=N_{j} \int p\left(u^{\prime} \mid u_{j}\right) p\left(x_{j} \ominus u^{\prime}\right) d u^{\prime},
\end{aligned}
$$

with $N_{i}$ and $N_{j}$ normalization factors. These lead to the following update for the single particle:

$$
x_{j}=\left(x_{j} \ominus\left(u_{i} \oplus n_{u_{i}}\right)\right) \oplus\left(u_{j} \oplus n_{u_{j}}\right),
$$

where $n_{u_{i}}$ and $n_{u_{j}}$ are samples taken by $p\left(u^{\prime} \mid u\right)$.

Note that if $\mathcal{R}_{i}$ and $\mathcal{R}_{j}$ do not communicate over a time interval $\left(t_{a}, t_{b}\right)$ (e.g., due to the fact that they are far from each other) the motion update of $\mathcal{R}_{j}$ is not performed. At $t_{b}$, when communication is resumed, $\mathcal{R}_{i}$ uses $v_{j}^{t_{b}} \ominus v_{j}^{t_{a}}$ as displacement for the motion update. This explains why the robots send out the total displacement $v_{j}^{t}$ rather than the last incremental displacement $u_{j}^{t}$. The outcome of the update step are the beliefs $\left\{\overline{\operatorname{bel}}\left(x_{j}^{t}\right)\right\}$ (see Fig. 2.)

The main difference with respect to the filter in [1] is in the measurement update. Since P-MultiBeaReg only produces relative bearing-orientation, the measurements have a lower dimension than the state. However, the generalization of the update rule is straightforward and given by

$$
p\left(x_{j} \mid \hat{\xi}_{j}\right)=N p\left(\hat{\xi}_{j} \mid x_{j}\right) p\left(x_{j}\right),
$$

with $N$ is another normalization factor. Equation (2) allows the computation of the posteriors $\left\{\operatorname{bel}\left(\mathrm{x}_{\mathrm{j}}^{\mathrm{t}}\right)\right\}$ depicted in Fig. 2 by using the the beliefs $\left\{\overline{\operatorname{bel}}\left(x_{j}^{t}\right)\right\}$ and the relative bearingorientation estimate given by P-MultiBeaReg.

\footnotetext{
${ }^{4}$ See [1] for a discussion about the use of a separate belief for each robot
} rather than a single joint belief. 
Standard devices can be applied to improve the performance of the filter. For example, the initial prior distribution can be generated using the first measurements. Also, it is advisable to reduce the frequency of the measurement update with respect to the motion update in order to guarantee the independence assumption for successive measurements.

\section{EXPERIMENTAL RESULTS}

We have implemented and tested the proposed mutual localization system on a team of 4 Khepera III robots, with the aid of the multi-robot software platform MIP ${ }^{5}$. Each robot is equipped with a Hokuyo URG-04LX laser sensor that provides range-bearing readings at $10 \mathrm{~Hz}$ within a $240^{\circ}$ field of view, thus leaving a $120^{\circ}$ blind zone behind the robot. The robot detector is a simple feature extraction module that inspects the laser scan, looking for the characteristic indentation caused by the small 'hat' mounted atop each robot. Only the bearing (not the distance) measurement coming from the detector has been used. Displacements are computed by odometric integration at $10 \mathrm{~Hz}$ to match the robot detector refresh rate. Synchronization among the robots is not an issue, essentially because the communication delay is not significant at the typical robot speeds. The robots move with a pseudo-random navigation strategy that incorporates obstacle avoidance.

Results from an experiment using 500 particles for each filter are shown in Fig. 4. The robots start in a square, ambiguous configuration in which the registration problem has multiple solutions (first snapshot). As a consequence, the filter particles are very sparse at the beginning, and there is no separation between the clouds associated to the different robots. When the robots start moving, the symmetry of the formation is broken, and P-MultiBeaReg is able to compute a single solution. Hence, the robot clouds starts to separate. Still, a small displacement does not allow the filters to recover an acceptable estimate of the scale, so that the particles are distributed over circular sectors (second snapshot). When the robots have moved enough for the filters to recover the scale (third and fourth snapshots), the results of P-MultiBeaReg are essentially used to update and improve the estimates.

For comparison, we have also run the localization method described in [1], whose results are obtained using the full position (distance plus bearing angle) measurements, and represent thus a sort of 'ground truth' for our new partiallyinformed localization system. Figure 5 plots the differences between the pose estimate of $\mathcal{R}_{1}$ computed by $\mathcal{R}_{4}$ using the two methods. There is a clear mismatch at the beginning of the experiment; however, it should be considered that the ambiguity also affects the fully informed method. As soon as the ambiguity is broken, the mismatch between the two methods becomes negligible.

Due to the small number of robots and to the limited field of view of the Hokuyo sensor, it happened frequently in the experiments that there was no triplet of robots 'seeing' each other. This is obviously a problem for P-MultiBeaReg. For

\footnotetext{
${ }^{5}$ See http://www.dis.uniroma1.it/labrob/software/MIP.
}

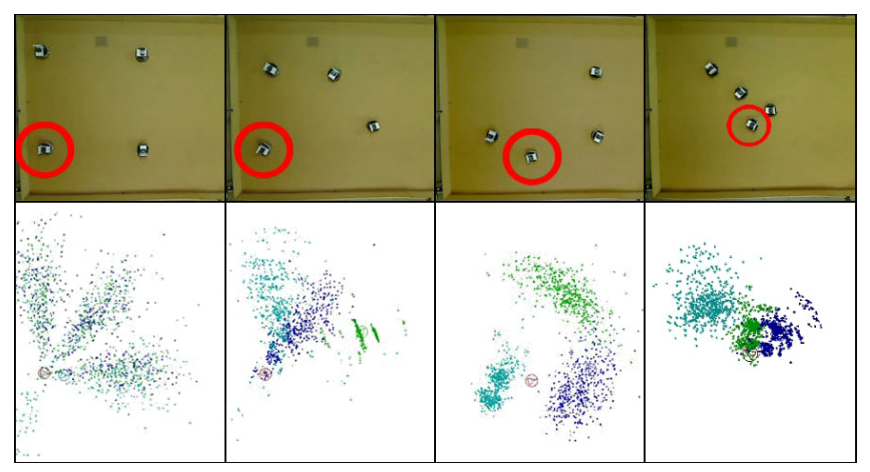

Fig. 4: Experiment. Top: Snapshots of the scene. Bottom: Sample distributions computed by $\mathcal{R}_{4}$ (circled).
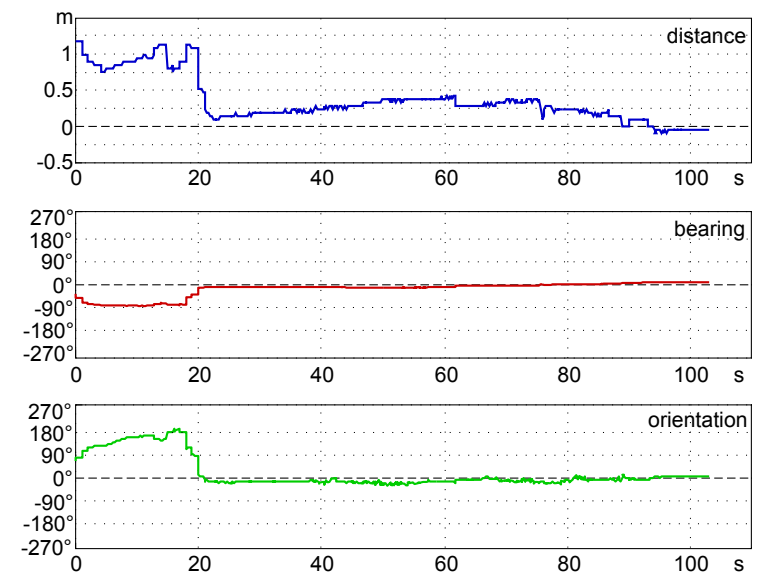

Fig. 5: Experiment: Differences between the pose estimate of $\mathcal{R}_{1}$ computed by $\mathcal{R}_{4}$ using the proposed method and the method in [1].

example, the growth of the distance error in Fig. 5 between $t=20$ and $t=40$ is due to this phenomenon.

In order to avoid the above difficulty, we have also tested the algorithm also in simulation with a $360^{\circ}$ field of view. The results are shown in Fig. 6, and the errors on the pose estimate of $\mathcal{R}_{1}$ computed by $\mathcal{R}_{4}$ are plotted in Fig. 7. In this case, a ground truth was obviously available from the simulation. As expected, the convergence is faster and the estimates are more precise than in the previous case. Fig. 7 shows also the errors for the best particle (i.e., the particle with the largest weight), which converges quickly as expected. In particular, when the simmetry is broken, this error reduces to zero immediately.

Video clips of both the experiment and the simulation are shown in the accompanying video. See also http://www.dis.uniroma1.it/labrob/research/mutLoc.html for additional material.

\section{CONCLUSIONS}

We have presented a decentralized method for mutual localization in multi-agent systems using anonymous bearing measurements. This is an extension of our previous work [1] on this topic, which assumed the availability of full position (i.e., including distance) measurements. The idea here is to achieve localization using non-depth, low-cost sensors like cameras in place of range finders. 


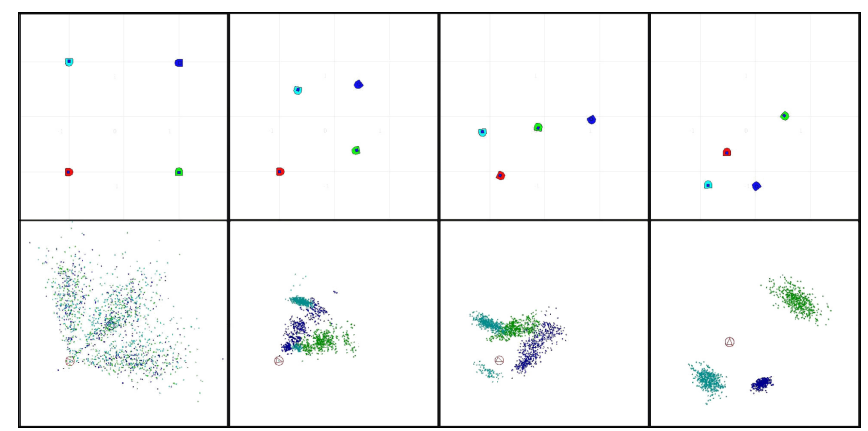

Fig. 6: Simulation. Top: Snapshots of the scene. Bottom: Sample distributions computed by $\mathcal{R}_{4}$ (red).
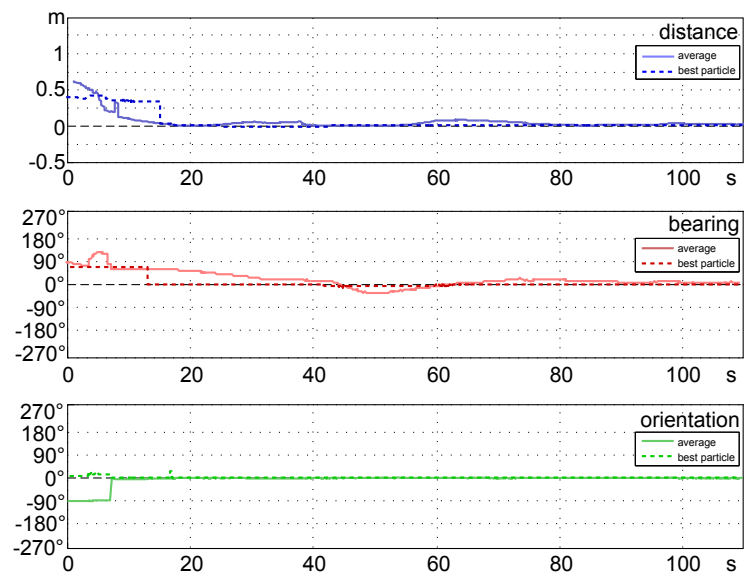

Fig. 7: Simulation: Errors on the pose estimate of $\mathcal{R}_{1}$ computed by $\mathcal{R}_{4}$. Dashed lines refer to the estimate of the best particle, solid lines refer to the estimate obtained by averaging the particles.

Experimental testing has shown that the localization system is able to reconstruct the missing distance information, leading to an overall performance that is still satisfactory. The price for the use of less-informative sensors is a slower convergence of the estimation error. This is essentially due to the fact that the algorithm needs significant robot displacements to recover the scaling factor, especially if the initial configuration is ambiguous due to symmetries.

Among the future developments of this work we mention:

- An analysis of the complexity of P-MultiBeaReg.

- Testing the mutual localization system with large numbers of robots. We expect the performance to improve significantly because P-MultiBeaReg will be able to identify and use many triangles, thus alleviating the ambiguity deriving from local symmetries.

- An extension to 3D agents (i.e, to $S E(3)$ ), for the application to swarms of flying robots.

\section{REFERENCES}

[1] A. Franchi, P. Stegagno, and G. Oriolo, "Probabilistic mutual localization in multi-agent systems from anonymous position measures," in 49th IEEE Conf. on Decision and Control, Atlanta, GA, Dec. 2010, pp. 6534-6540.

[2] R. Olfati-Saber, J. A. Fax, and R. M. Murray, "Consensus and cooperation in networked multi-agent systems," Proceedings of the IEEE, vol. 95, no. 1, pp. $215-233,2007$.

[3] H. G. Tanner, A. Jadbabaie, and G. J. Pappas, "Flocking in fixed and switching networks," IEEE Trans. on Automatic Control, vol. 52, no. 5, pp. $863-868,2007$
[4] A. Howard, L. E. Parker, and G. S. Sukhatme, "Experiments with a large heterogeneous mobile robot team: Exploration, mapping, deployment and detection," International Journal of Robotics Research, vol. 25, no. 5-6, pp. 431-447, 2006.

[5] N. Michael and V. Kumar, "Planning and control of ensembles of robots with non-holonomic constraints," International Journal of Robotics Research, vol. 28, no. 8, pp. 962-975, 2009.

[6] P. Yang, R. A. Freeman, G. J. Gordon, K. M. Lynch, S. S. Srinivasa, and R. Sukthankar, "Decentralized estimation and control of graph connectivity for mobile sensor networks," Automatica, vol. 46, no. 2, pp. 390-396, 2010.

[7] P. Yang, R. A. Freeman, and K. M. Lynch, "Multi-agent coordination by decentralized estimation and control," IEEE Trans. on Automatic Control, vol. 253, no. 11, pp. 2480-2496, 2008

[8] J. Fink, N. Michael, S. Kim, and V. Kumar, "Planning and control for cooperative manipulation and transportation with aerial robots," International Journal of Robotics Research, vol. 30, no. 3, 2010.

[9] M. Schwager, B. J. Julian, and D. Rus, "Optimal coverage for multiple hovering robots with downward facing cameras," in 2009 IEEE Int. Conf. on Robotics and Automation, Kobe, Japan, May 2009, pp. 35153522.

[10] L. C. A. Pimenta, V. Kumar, R. C. Mesquita, and G. A. S. Pereira, "Sensing and coverage for a network of heterogeneous robots," in 47th IEEE Conf. on Decision and Control, Cancun, Mexico, Dec. 2008, pp. 3947-3952.

[11] J. Aspnes, T. Eren, D. K. Goldenberg, A. S. Morse, W. Whiteley, Y. R. Yang, B. D. O. Anderson, and P. N. Belhumeur, "A theory of network localization," IEEE Trans. on Mobile Computing, vol. 5, no. 12, pp. $1663-1678,2006$

[12] T. Eren, W. Whiteley, and P. Belhumeur, "Using angle of arrival (bearing) information in network localization," in 45th IEEE Conf. on Decision and Control, San Diego, CA, Jan. 2006, pp. 4676-4681.

[13] G. Piovan, I. Shames, B. Fidan, F. Bullo, and B. Anderson, "On frame and orientation localization for relative sensing networks," in 47th IEEE Conf. on Decision and Control, Cancun, Mexico, Dec. 2008 , pp. 2326-2331.

[14] J. Fang and A. S. Morse, "Network localization using graph decomposition and rigidity," in 48th IEEE Conf. on Decision and Control, Dec. 2008, pp. 1091-1096.

[15] I. Shames, B. Fidan, and B. D. O. Anderson, "Minimization of the effect of noisy measurements on localization of multi-agent autonomous formations," Automatica, vol. 45, no. 4, pp. 1058-1065, 2009.

[16] D. Fox, W. Burgard, H. Kruppa, and S. Thrun, "A probabilistic approach to collaborative multi-robot localization," Autonomous Robots, vol. 8, no. 3, pp. 325-344, 2000 .

[17] S. I. Roumeliotis and G. A. Bekey, "Distributed multirobot localization," IEEE Trans. on Robotics, vol. 18, no. 5, pp. 781-795, 2002

[18] A. Howard, M. J. Matarić, and G. S. Sukhatme, "Localization for mobile robot teams using maximum likelihood estimation," in 2002 IEEE/RSJ Int. Conf. on Intelligent Robots and Systems, Lausanne, Switzerland, Sep. 2002, pp. 434-439.

[19] A. Das, J. Spletzer, V. Kumar, and C. Taylor, "Ad hoc networks for localization and control," in 41th IEEE Conf. on Decision and Control, Dec. 2002, pp. 2978-2983.

[20] A. I. Mourikis and S. I. Roumeliotis, "Performance analysis of multirobot cooperative localization," IEEE Trans. on Robotics, vol. 22 , no. 4, pp. 666-681, 2006.

[21] A. Franchi, G. Oriolo, and P. Stegagno, "Mutual localization in a multirobot system with anonymous relative position measures," in 2009 IEEE/RSJ Int. Conf. on Intelligent Robots and Systems, St. Louis, MO, Oct. 2009, pp. 3974-3980.

[22] — "On the solvability of the mutual localization problem with anonymous position measures," in 2010 IEEE Int. Conf. on Robotics and Automation, Anchorage, AK, May 2010, pp. 3193-3199.

[23] A. Martinelli, F. Pont, and R. Siegwart, "Multi-robot localization using relative observations," in 2005 IEEE Int. Conf. on Robotics and Automation, Barcelona, Spain, Apr. 2005, pp. 2797-2802.

[24] X. S. Zhou and S. Roumeliotis, "Determining the robot-to-robot 3d relative pose using combinations of range and bearing measurements (part II)," in 2011 IEEE Int. Conf. on Robotics and Automation, Shanghai, China, May. 2011, pp. 4736-4743.

[25] A. Martinelli and R. Siegwart, "Observability analysis for mobile robot localization," in 2005 IEEE/RSJ Int. Conf. on Intelligent Robots and Systems, Edmonton, Canada, Aug. 2005, pp. 1471-1476. 